\title{
6 (In-)Definitheit impliziter Argumente
}

Seit geraumer Zeit werden implizite Argumente in der Forschungsliteratur zur Argumentweglassung in sog. indefinite und definite (vgl. u. a. Fillmore 1986) bzw. gleichbedeutend in existentielle bzw. anaphorische Argumente (vgl. Condoravdi/ Gawron 1996) unterteilt. Nach Ansicht der Autoren,

the particular verb determines whether its IO [implicit object, TvdB] will be in one category or another [...] and they propose diagnostics to determine whether a verb selects indefinite (existential) or definite (anaphoric) IOs.

(Glass 2014: 128)

Im Folgenden werden die mit dieser Unterscheidung verbundenen zentralen Ideen sowie die jeweils vorgeschlagenen Verfahren zur Unterscheidung zwischen indefiniten und definiten impliziten Ergänzungen vorgestellt und kritisch reflektiert ${ }^{223}$. Dabei wird sich zeigen, dass die Unterscheidung zwischen beiden Gruppen impliziter Argumente mit verschiedenen Problemen behaftet ist, weshalb auch keines der vorgeschlagenen diagnostischen Verfahren zu eindeutigen Ergebnissen führt (vgl. ebd.). Vor allem die Analyse indefiniter Argumente bereitet erhebliche Schwierigkeiten, weshalb Junker et al. (2006: 8) sogar die Frage aufwerfen, inwieweit die Annahme von Verben - konkreter Verblesarten ${ }^{224}$ - mit indefiniten impliziten Argument überhaupt sinnvoll ist.

Die Notwendigkeit der Unterscheidung zwischen indefiniten und definiten impliziten Argumenten soll hier hinterfragt werden - insbesondere vor dem Hintergrund der in F2 formulierten Forschungsfrage sowie den im Zusammenhang mit semantischen Spezialisierungen impliziter Argumente gemachten Beobachtungen (vgl. Kapitel 5). Abweichend zu den Darstellungen in Kapitel 5 erfolgen die nachfolgenden Untersuchungen nicht einzelverbbezogen. Vielmehr wird ausgehend von Ruppenhofers Vorhersage, dass alle Verben derselben lexikalischen Gruppe semantische Rollen des gleichen Interpretationstyps unrealisiert lassen können, sofern sie Weglassung grundsätzlich zulassen (vgl. Ruppenhofer 2004; 2018), eine verbübergreifende Hypothese entwickelt. Zwar beziehen sich Ruppenhofers Vorhersagen vorwiegend auf englische Daten ${ }^{225}$; zuletzt konnte er

223 Die folgenden Darstellungen sollen die zentralen Entwicklungslinien der Unterscheidung zwischen indefiniten und definiten impliziten Argumenten aufzeigen; ein Anspruch auf Vollständigkeit aller in der Literatur vorfindbaren Ansätze wird dabei nicht erhoben.

224 Junker et al. weisen hier - m. E. zu Recht - auf die Notwendigkeit einer Lesartendifferenzierung hin; tatsächlich umgesetzt wird dieser Gedanke jedoch auch von ihnen nicht, wie Formulierungen der Art „Verbs like ,eat““ (Junker et al. 2006: 9) erkennen lassen.

225 Vgl. hierzu auch Ruppenhofer/Michalis (2014), die für das Englische davon ausgehen, dass wenn ,a particular frame element role is lexically omissible under a particular interpretation

Ә Open Access. ( 2021 Tanja von der Becke, publiziert von De Gruyter. (c) BY Dieses Werk ist lizensiert unter einer Creative Commons Namensnennung 4.0 International Lizenz.

https://doi.org/10.1515/9783110726268-006 
jedoch nachweisen, dass die für das Englische beobachteten Regularitäten sich auch auf deutsche Verben übertragen lassen (vgl. Ruppenhofer 2018: 211) ${ }^{226}$.

\subsection{Forschungsstand}

Als einer der ersten merkt Fillmore (1971b) an, dass „for certain predicates, silence (,zero') can replace one of the argument-expressions just in case the speaker wishes to be indefinite or non-committal about the identity of the argument“. Bei anderen Prädikaten ,silence can replace one of the argument-expressions just in the case the LS [locutionary source, TvdB] believes that the identity of the argument is already known by the LT [locutionary target, TvdB]“ (ebd. 380) ${ }^{227}$.

1986 greift Fillmore diese Unterscheidung erneut auf und schreibt, dass eine Unterscheidung zwischen indefiniten und definiten impliziten Argumenten notwendig ist (vgl. Fillmore 1986: 96). Bei definit impliziten Argumenten muss das, was weggelassen wird, aus dem Kontext ${ }^{228}$ herleitbar sein (vgl. ebd.); das impli-

(either anaphoric or existential) for one LU [lexical unit, TvdB] in a frame, then for any other LUs in the same frame that allow the omission of this same FE [frame element, TvdB], the interpretation of the missing frame element is the same“ (ebd. 66).

Entsprechende Hypothesen werden ausgehend von dem englischsprachigen FrameNet einen framebasierten Online-Thesaurus - formuliert. FrameNet wird seit 1997 am International Computer Science Institute (ICSI) in Berkeley nach den Prinzipien der Fillmore'schen Frame-Semantik entwickelt. Die zentrale Idee dabei ist, dass die Bedeutung der meisten Wörter auf Basis semantischer Frames verstanden werden kann (vgl. FrameNet 2015). Ein semantischer Frame ist „eine konzeptuelle Struktur zur Beschreibung eines spezifischen Situationstyps, Objekttyps oder Ereignistyps mit seinen jeweilig verknüpften Partizipanten und Eigenschaften“ (Kunze/Lemnitzer 2007:142). So involviert der Frame Ingestion als Rollen u.a. die FrameElemente (FE) Ingestibles und Ingestor, und wird durch Verben wie breakfast, consume, devour, dine, down, drink und eat aktiviert. Letztere bilden aufgrund ihrer frameauslösenden Eigenschaft die Basiseinheit von Frame-Net.

226 Eine Übertragung von FrameNet auf das Deutsche ist nicht ohne weiteres möglich, da die Bedeutungen deutscher Verben sich nicht immer auf die auf englischen Daten basierenden Frame-Unterscheidungen abbilden lassen (vgl. Burchardt et al. 2009: 225). Vor diesem Hintergrund greift Ruppenhofer (2018) auf SALSA, eine framebasierte lexikalisch-semantische Ressource, die die Besonderheiten des Deutschen - u. a. durch Ergänzung fehlender Frames sowie die Berücksichtigung sprachspezifischer Lexikalisierungsmuster - beachtet, zurück. Da die Datengrundlage von SALSA allerdings nicht annähernd so groß ist wie die von FrameNet, bedient sich Ruppenhofer zusätzlich einzelner Beispiele des Huge German Corpus (vgl. Ruppenhofer 2018: 210)

227 „The producer of a speech act will be called the locutionary source (LS), the addressee will be referred to as the locutionary target.“ (Fillmore 1971b: 371)

228 Problematisch an Fillmores Darstellungen ist, dass nicht eindeutig angegeben wird, was genau er unter Kontext versteht. Dieser Umstand schwächt nach Németh' Ansicht Fillmores 
zite Argument ist damit eine definite Entität, die aus dem (sprachlichen) Kontext identifizierbar ist (vgl. Junker et al. 2006: 3). Bei indefinit impliziten Argumenten hingegen ist der Referent des impliziten Arguments unbekannt oder - auf eine bestimmte Art und Weise - unwichtig (vgl. Fillmore 1986: 96); das implizite Argument ist indefinit (vgl. Junker et al. 2006: 3). Die nachfolgenden Beispiele veranschaulichen diese Unterscheidung: In (403) und (404) wird etwas Konkretes, aus dem (sprachlichen) Kontext Herleitbares herausgefunden bzw. gefolgt (vgl. ebd.).

(403) Samir found out at midnight. (ebd.)

(404) Hershad followed quietly. (ebd.)

Anders verhält es sich in (405) und (406); hier wird irgendetwas gegessen bzw. gesungen; die konkrete Identität der jeweils unrealisierten Entität wird dabei nicht thematisiert (vgl. ebd.):

(405) Omar eats at midnight. (ebd.)

(406) Kahlid sings at midnight. (ebd.)

Fillmore entwickelt einen lexikalisch basierten Ansatz, um das Phänomen der Argumentweglassung zu erfassen. Auch die Unterscheidung von definiten und indefiniten impliziten Argumenten ${ }^{229}$ stellt für ihn eine fest zum Verb gehörende Eigenschaft dar, die es erforderlich macht, für jedes $\operatorname{Verb}^{230}$ anzugeben, welches seiner

\footnotetext{
Argumentation gegen pragmatische Einflussfaktoren deutlich ab: „Fillmore does not define explicitly what he means by context. The analyses of his examples may indicate that the notion of context is understood by him very narrowly and that he takes into account only what is called the linguistic context, i. e. the utterance-context in which the implicit argument occurs, or, rarely, the preceding discourse. Unfortunately, one cannot find any preceding discourse part in the explanation of his examples, and this reduces the convincing force of the examples to a large extent. “ (Németh 2010: 167)
}

229 Einige Verben weisen nach Ansicht von Fillmore sowohl definite als auch indefinite implizite Argumente auf: In Sätzen wie I contributed five dollars to the movement kann sowohl die Art der Spende implizit bleiben, wobei I contributed to the movement gleichbedeutend ist mit I contributed something to the movement, als auch der Empfänger der Spende, wobei I contributed five dollars nur interpretierbar ist, wenn der Empfänger der Spende aus dem Kontext hergeleitet werden kann (vgl. Fillmore 1986).

230 Fillmore merkt in diesem Zusammenhang bereits an, dass das „DNC phenomenon is restricted to particular lexical items [...]. For polysemous items, DNC may be restricted to particular senses.“ (ebd. 99) Somit verweist bereits Fillmore darauf, dass - im Sinne der hier fomulierten 
Komplemente bei Weglassung indefinit bzw. definit interpretiert werden muss (vgl. Fillmore 1986: 98). Rein semantische und rein pragmatische Erklärungsversuche schließt Fillmore kategorisch aus: Während ein (rein) semantischer Erklärungsansatz seiner Auffassung nach aufgrund von synonymen Verben, die sich hinsichtlich ihrer Argumentweglassungsbedingungen unterscheiden, unbrauchbar zur Erfassung der Unterscheidung definiter und indefiniter impliziter Argumente ist, hält Fillmore einen (rein) pragmatischen Erklärungsversuch für unmöglich, da es zahlreiche Verben gibt, die unabhängig davon, wie komplex der gegebene (sprachliche) Kontext ist, eine Weglassung ihrer Komplemente kategorisch ausschließen (z. B. I locked) (vgl. Scott 2006: 141).

Nach Fillmore ist ein indefinit implizites Argument vergleichbar mit einer explizit realisierten indefiniten Nominalphrase ${ }^{231}$ : „[I]t is markedly indefinite, by which I mean that it is obligatorily disjoint in reference with anything saliently present in the pragmatic context“ (Fillmore 1986: 97). Hieraus ergibt sich, dass die Vorerwähntheit des wegzulassenden Arguments in entsprechenden Fragekontexten bei Verben mit indefinit implizitem Argument ${ }^{232} \mathrm{zu}$ unnatürlichen Strukturen führt. So kann What happened to my sandwich? - Fido ate! keiner wohlgeformten Konversation entsprechen (vgl. ebd.).

Definit implizite Argumente zeichnen sich hingegen durch ihr Potenzial einer kontextuell definiten Interpretation aus (vgl. ebd.); entsprechend bereitet die Vorerwähntheit des wegzulassenden Arguments bei diesen Verben keine Probleme, wie das nachfolgende Beispiel zeigt: The government had a secret plan. If they aren't careful, the media will find out (vgl. AnderBois 2012: 44).

Forschungsfrage F2 - ,it is not „the verb“ so much as ,the verb-on-a-sense“ that seems to permit/ prohibit null complements“ (Junker et al. 2006: 5).

231 Fillmores Annahme, wonach indefinit implizite Argumente sich gleich/ähnlich verhalten, wie syntaktisch realisierte indefinite Nominalphrasen, wird in der Literatur als nicht unproblematisch angesehen. Glass (2014: 128f.) etwa weist in diesem Zusammenhang darauf hin, dass „[i]ndefinites can antecede pronouns [...]. Thus, indefinite IOs should license pronouns as well. But this prediction does not [...] hold“, wie die Beispiele in (XV) zeigen:

(XV) a. A sailor walked in. She sat down. (ebd.)

b. Mark lifted. ?They were heavy. (ebd.)

Entsprechend konstatiert sie, dass „,indefinite‘ IOs do not behave like regular indefinites with respect to pronouns“ (ebd.).

232 Indefinite implizite Argumente treten nach Fillmore in zwei verschiedenen Arten auf: „one involving a semantic object of considerable generality, the other requiring the specification of various degrees of semantic specialization“ (ebd. 96). Ausführliche Darstellungen zu dieser Unterscheidung finden sich in Kapitel 5.2. 
Ausgehend von der (kontextuellen) Herleitbarkeit impliziter Elemente (vgl. Velasco/Munoz 2002: 8) schlägt Fillmore als Testverfahren zur Unterscheidung zwischen definiten und indefiniten impliziten Argumenten den admission of ignorance test ${ }^{233}$ vor. Hierbei wird überprüft,

whether it would sound odd for a speaker to admit ignorance of the identity of the referent of the missing phrase. It's not odd to say things like, "He was eating; I wonder what he was eating"; but it is odd to say things like "They found out; I wonder what they found out." The missing object of the surface intransitive verb EAT is indefinite; the missing object of the surface-intransitive verb FIND OUT is definite.

(Fillmore 1986: 96)

In ähnlicher Weise argumentiert auch Shopen (1973) und führt zur Unterscheidung indefiniter bzw. definiter impliziter Argumente den sog. natural-sequencetest ${ }^{234}$ durch. Bei indefiniten Weglassungen wie in (407a) führt die Frage des zweiten Sprechers zu einer natürlichen Sequenz, da sie nicht im Widerspruch zu der vom ersten Sprecher gemachten Aussage steht (vgl. Ebd. 67). Die Frage What did he refuse to do? bei definiter Weglassung in (407b) führt dagegen zu einer unnatürlichen Sequenz, da sie gegen die Voraussetzung der Definitheit verstößt (vgl. ebd. 68):

(407) a. Bill received a letter today. - Who did he get it from?

b. Bobby refused.

- ${ }^{\star}$ What did he refuse to do?

Auch andere Autoren weisen vor dem Hintergrund kontextueller Rekonstruierbarkeit auf die Unterscheidung zwischen definiten und indefiniten impliziten Argumenten hin. Allerton unterscheidet zwischen definiten und indefiniten impliziten Argumenten, indem er angibt, dass bei definit impliziten Argumenten etwas weggelassen wird, das eindeutig aus dem sprachlichen oder situativen Kontext hergeleitet

233 Scott (2006: 141) bezeichnet Fillmores Test als „diagnostic ,wonder“ test“. Er basiert auf der Annahme, dass man sich nicht über etwas wundern kann, das man bereits weiß .

234 Der von Shopen vorgeschlagene natural sequence test ist nicht unproblematisch. So hängt die Beurteilung, ob eine Frage-Antwort-Sequenz als natürlich oder unnatürlich empfunden wird, maßgeblich vom Beurteilenden selber ab. Denn „seine Vorstellungskraft und sein Wissen um normale Kommunikationssituationen entscheiden wesentlich darüber, ob er es für plausibel hält, dass die Behauptungssequenz genügend informationsgesättigt ist, um als inhaltlich abgeschlossene und zugleich diskurseinleitende Äusserungseinheit gelten zu können“ (Dentler 1990: 45).

Dieser Einwand bedeutet zwar nicht, dass der Test zu keinerlei Ergebnissen führt; er soll nur zeigen, „dass der Test von begrenzter Tragweite ist, weil er [...] auf ganz bestimmten Verben und ihrem elliptischen Gebrauch in ganz besonderen Kommunikationssituationen basiert“ (ebd.: 46). 
werden kann, während wir bei indefinit impliziten Argumenten mit etwas kontextuell nicht Herleitbarem konfrontiert werden (vgl. Allerton 1975: 214). Zur Unterscheidung $^{235}$ schlägt Allerton zwei unterschiedliche Frage-Antwort-Sequenzen vor:

(408) What did you do this afternoon?

I just read/sewed/typed.

${ }^{\star}$ I just looked/watched/pushed.

(409) I see you've got today's ,Guardian“.

May I look?

*May I read?

In (408), ,the indefinite nature of the question only permits indefinite deletion in the answer“ (ebd. 215), wohingegen in (409) „the presence of the contextual item today's' Guardian', a likely object for look at or read, means that only contextual [definite, TvdB] deletion is possible.“ (ebd. 215)

Auch Thomas (1979) unterscheidet zwischen definiten und indefiniten impliziten Argumenten. Den Unterschied zwischen ellipsis (entspricht den definit impliziten Argumenten) und nonrealization (entspricht den indefinit impliziten Argumenten) sieht er in der Differenz zwischen obligatorischen und optionalen Elementen (vgl. ebd. 52). Zur Unterscheidung dieser Dichotomie schlägt er eine Frage-Antwort-Sequenz mit ausweichender Zurückweisung vor. Je nachdem, ob die elliptische Verbverwendung in der Antwort als angemessen oder unangemessen $\mathrm{zu}$ bewerten ist, wird die nicht realisierte Argumentstelle als indefinit oder definit interpretiert:

(410) A: Have you been eating onions?

B: I've been eating, but not onions.

(411) A: Do you expect to pass your driving test?

B: ${ }^{\star}$ I expect to pass, but not my driving test.

235 Allerton merkt an, dass die Unterscheidung zwischen definiten und indefiniten impliziten Argumenten nicht immer so klar ist, wie sie sein sollte (vgl. Allerton 1975: 217). Dieses Problem resultiert seiner Auffassung nach u.a. aus den häufig beobachtbaren sortalspezifischen Interpretationen der argumentlosen Sätze: So erlauben zahlreiche Verben zwar eine indefinite Weglassung, unterliegen dabei jedoch häufig einer semantischen Spezialisierung. (vgl. ebd.) Ausführliche Darstellungen hierzu finden sich in Kapitel 5.2. 
Die Frage-Antwort-Sequenz in (410) ist in Ordnung, da ,its first part, being a case of nonrealization, does not require that the particular element of the proceeding sentence for which it had potential be ,understood'. Its second part is, in this way, not inconsistent with the first.“ (ebd. 57). Dagegen erscheint die Frage-Antwort-Sequenz in (411) problematisch, „because their first parts, being elliptical, require that the relevant particular elements of the preceding sentences be ,understood'. It follows, then, that their second parts contradict their first parts.“ (ebd.).

Sæbø (1984) plädiert ebenfalls für eine grundsätzliche Zweiteilung in indefinit-fakultative Ergänzungen, die strenggenommen nicht vom Kontext abhängen, und definit-fakultative Ergänzungen, die in einem strengen Sinne kontextbestimmt sind (ebd. 97). Er führt zahlreiche authentische Beispiele für (in-)definite implizite Argumente im Deutschen auf (Sæbø 2003: 816). Dabei gilt: Verwendet man Fakultativität bei indefinit-fakultativen Verben,

begnügt man sich [...] mit dem Verb, ja so spezifiziert man eben nichts darüber hinaus: man läßt es offen, um was für ein >Objekt< es sich gerade handelt. Ganz, wie wenn man die Stelle mit etwas, jemand (im Kasus und eventuell mit Präposition) besetzt hätte.

(Sæbø 1984: 100)

In anderen Fällen, den sog. definit-fakultativen Verben,

müssen wir eine Anapher hinzudenken. Der Mitspieler ist kontextbestimmt, und wir können sagen, daß im Weglaßfall eine Anapher eingebaut ist, im Verb (oder sonstigen Valenzträgern). [...] Ohne geeigneten Kontext ist der nicht gefüllte Satz nicht ganz interpretierbar. ${ }^{236}$

(ebd. 101)

Im Sinne dieser Bedeutungsrelationen geht Sæbø davon aus, dass bei impliziten Argumenten je nach Verb „entweder eine durch Existenzquantor gebundene oder freie Variable [...] mitverstanden wird“ (ebd. 99).

Als Test zur Differenzierung zwischen indefiniten und definiten impliziten Argumenten nennt Sæbø das Kriterium der situationsfreien Interpretation; dabei geht es um die Frage, ob ein Satz mit implizitem Argument leicht als inhaltlich abgeschlossen interpretiert werden kann - dann liegt eine indefinit-fakultative

236 Einschränkend merkt Sæbø an: „Bei einigen Verben muß die fehlende Ergänzung nicht vorerwähnt sein. Dann ist es nicht treffend, von eingebauter Anapher zu reden. Kontextbestimmt ist die [...] Ergänzung noch, doch wir müssen den Kontextbegriff ausweiten und von Äußerungs-, Situationskontext sprechen. Es handelt sich um eine Art von Deixis. [...] Die ,Referenz‘ ist nicht immer zum Greifen nah, sie kann etwas zurückliegen. Man bezieht sich manchmal implizit auf Wahrgenommenes, Beobachtetes, Erlebtes, Erfahrenes; auf die Situation und ihre Vorgeschichte; auf background knowledge.“ (Sæbø 1984: 106) 
Ergänzung vor (z. B. Er isst) - oder ob eine entsprechende Interpretation ausgeschlossen/schwierig ist - in diesen Fällen liegt eine definit-fakultative Ergänzung vor (z. B. Er lehnt $a b)$.

Auf die von Sæbø genannten unterschiedlichen Paraphraserelationen indefiniter und definiter impliziter Argumente weisen auch andere Autoren hin. Engelberg (2002: 388) gibt ebenfalls für das Deutsche an, dass der Umstand, dass ein implizites Argument nicht durch ein Indefinitpronomen ersetzt werden kann, den Eindruck stärkt, dass das implizite Argument definit interpretiert werden muss. Shopen (1972; 1973) konstatiert für das Englische, dass in Sätzen mit indefiniter Weglassung (z. B. Bill got a letter today) der Referent des impliziten Arguments weder Sprecher noch Hörer bekannt sein muss - das implizite Argument (hier der Absender des Briefs) kann also als Präpositionalphrase mit Indefinitpronomen realisiert werden (z. B. Bill received a letter from someone); dagegen muss der Referent des impliziten Arguments in Sätzen mit definiter Weglassung (z. B. Bobby refused) im gemeinsamen Fokus von Sprecher und Hörer liegen - weshalb das nicht realisierte Argument nicht durch ein Indefinitpronomen, sondern durch ein Definitpronomen wie it realisiert werden muss (z. B. Bobby refused to do it) .

In ähnlicher Weise argumentiert Allerton (1975); er gibt an, dass die beiden Weglassungstypen mit unterschiedlichen Proformen korrelieren: Während definite fakultative Ergänzungen durch definite Proformen (z. B. him, her, it, them, this, these, that, those) ersetzt werden können, lassen sich indefinit fakultativen Ergänzungen indefinite Proformen zuweisen (z. B. something, someone, somebody, people, things).

Auf die mit definiten und indefiniten impliziten Argumenten verbundenen unterschiedlichen Bedeutungsrelationen weist Groefsema (1995) hin, indem sie feststellt, dass

there is a difference between INC verbs such as eat, drink and read, and DNC verbs, such as win and follow in that INC verbs only put selection restrictions on their internal argument as far as the type of THING that is at stake, while DNC verbs specify that an instance of a THING is at stake.

(ebd. 147)

Von vielen Wissenschaftlern (vgl. Groefsema 1995: 142; Junker et al. 2006: 6ff.; Jacobs 1994b: 298; Ágel 2000: 257; Härtl 2008: 117) kritisch betrachtet wird insbesondere die in allen bisher genannten Ansätzen vertretene Auffassung, dass bei indefinit impliziten Argumenten im Unterschied zu definiten die Indentität des weggelassenen Komplements unbekannt, unwichtig oder sogar beides ist (vgl. Scott 2006: 141). Zwar weisen auch sie auf den Unterschied entsprechender Verwendungsweisen hin, geben dabei jedoch an, dass insbesondere die Beschreibung indefinit impliziter Argumente stark vereinfacht ist (vgl. Engelberg 2002: 401). 
In diesem Sinne merkt z. B. Jacobs (1993b: 13) für das Deutsche an, dass Sätze mit einem definit impliziten Argument wie dass Peter akzeptiert nicht nur bedeuten, dass Peter etwas Bestimmtes akzeptiert; vielmehr bedeutet es, dass er etwas bestimmtes, aus dem (sprachlichen) Kontext Identifizierbares akzeptiert. Anders verhält es sich dagegen bei Sätzen mit scheinbar indefinit implizitem Argument wie dass Peter heiratet - hier müssen Sprecher und Hörer nicht wissen, wen Peter heiraten wird. Da das Verb heiraten aber neben Sätzen, in denen es im Sinne von jemanden heiraten verstanden wird, auch in solchen Sätzen Verwendung findet, in denen „das implizite Objekt von den Diskursteilnehmern identifiziert werden kann, weshalb es hier nicht mit jemanden versprachlicht werden kann“ (Jacobs 1994b: 298), vgl. (412a) vs. (412b), hält Jacobs den Terminus indefinit für zu unpräzise:

(412) a. Ich weiß nicht, warum heutzutage noch irgendwer heiratet. (ebd.)

b. Peter ist schon lange mit Gerda verlobt, aber heiraten will er erst, wenn Gerda Professorin ist. (ebd.)

Hinsichtlich der Eingeführtheit in den Diskurs steht Definitheit ${ }^{237}$ seiner Auffassung nach daher nicht Indefinitheit, sondern vielmehr Definitheitsneutraliät gegenüber (vgl. ebd. 301). In diesem Sinne zeichnen sich die impliziten Argumente von Verben wie heiraten dadurch aus, dass sie „,weder fordern noch verbieten, daß ihr Referent bereits in den Diskurs eingeführt worden ist“ (ebd. 298). Der Unterschied zwischen definiten und indefiniten (bzw. definitheitsneutralen) impliziten Argumenten besteht demnach darin, dass man beispielsweise sagen kann Er heiratet, aber ich weiß nicht, wen, aber nicht 'Er akzeptiert, aber ich wei $\beta$ nicht, was (vgl. ebd. 300).

Zur Unterscheidung beider Realisierungsformen zieht Jacobs den sog. Negationstest heran: Der Unterschied zwischen entsprechenden impliziten Argumenten wird seiner Meinung nach bei Negation deutlich: Die Negation daß Peter nicht heiratet bedeutet, dass er niemanden heiratet (vgl. Jacobs 1993b: 13). Das schließt seiner Auffassung nach nicht aus, dass man den Satz Er heiratet nicht auch ,in Situationen verwendet, in denen bekannt ist, wen Peter heiraten wollte“ (Jacobs 1994b: 300 - FN 43). Im Unterschied dazu bedeutet die Negation daß Peter nicht akzeptiert, dass es etwas kontextuell Herleitbares gibt, dass Peter akzeptiert (vgl. Jacobs 1993b: 13).

$237 \mathrm{Zu}$ den definit impliziten Argumenten zählt Jacobs auch die reflexiven impliziten Ergänzungen, deren Referenten identisch mit dem des Subjekts und damit schon eingeführt sind (vgl. Jacobs 1994b, S. 301 - FN 50). 
Auch Ágel (2000) weist in Anlehnung an Jacobs darauf hin, dass der Begriff indefinit nicht präzise genug ist. Er greift deshalb Jacobs' Gegenüberstellung definit vs. definitheitsneutral auf, zieht jedoch die seines Erachtens „passendere begriffliche Opposition (diskurs)ungebunden/(diskurs)gebunden“ vor ${ }^{238}$ (ebd. 257).

Als Test zur Unterscheidung entsprechender impliziter Argumente schlägt Ágel den (Diskurs-)Bindungstest vor: In Sätzen wie Er heiratet, aber ich weiß nicht, wen, ist das implizite Argument ungebunden, d. h. definitheitsneutral, weshalb der Aber-Anschluss normal erscheint. Das implizite Argument in Sätzen wie Er willigt ein, aber ich weiß nicht, in was ist dagegen gebunden, d. h. definit, weshalb der Aber-Anschluss hier nicht normal ist.

Engelberg (2002) schließt sich mit Verweis auf entsprechende Beispiele (vgl. 413) ebenfalls dieser Beobachtung an, indem er feststellt: „Although a non-definite implicit argument $\mathrm{x}^{-\mathrm{d}}$ is interpreted indefinitely in most cases, it allows definite interpretation, too. Thus it can be said to be neutral with respect to definiteness and might better be represented as $\mathrm{x}^{ \pm \mathrm{d} \text { ، }}(\mathrm{ebd} .401)$ :

(413) a. als ich ins Zimmer kam, saß sie im Sessel und laß (indefinit) (ebd.)

$a^{\prime}$. sie nahm den neuen Roman von Grass und las (definit) (ebd.)

b. er saß den ganzen Abend vorm Fernseher und strickte (indefinit) (ebd. 402)

$b^{\prime}$. weil er den Pullover schnell fertig haben wollte, setzte er sich hin und strickte (definit) (ebd.)

Auch in der englischsprachigen Literatur finden sich Angaben, wonach die Annahme für indefinite Argumente, dass ,the understood argument [...] is obligatorily disjoint in reference with anything saliently present in the context“ (Groefsema 1995: 142), nicht immer zutrifft. Groefsema konstatiert z. B. für das Verb eat, dass ein Satz wie John brought the sandwiches and Ann ate nur so interpretiert werden kann,

that what Ann ate were the sandwiches that John brought, although it leaves unspecified how many of the sandwiches Ann ate. This means that although the understood argument does not necessarily refer to all the sandwiches that John brought, neither it is disjoint in reference with the sandwiches John brought.

(ebd.)

238 „Der Begriff ,(Diskurs)Ungebundenheit‘ entspricht dabei faktisch Sæbøs ,Gebundenheit durch Existenzquantor““ (Ágel 2000: 257). Hierdurch, so Ágel, werden die Nichtrealisierungsphänomene nicht logisch, sondern pragmatisch behandelt. Außerdem präjudiziert die neue Gegenüberstellung seiner Auffassung nicht, dass zwei Grundtypen von Nichtrealisierungsphänomenen bereits im Lexikoneintrag der Verben verankert sind. 
Trotz dieser Beobachtungen findet eine terminologische Anpassung hier nicht statt.

AnderBois (2012) hingegen greift die Unterscheidung zwischen definiten und indefiniten impliziten Argumenten zunächst auf, ergänzt sie anschließend jedoch um eine weitere Gruppe, die sog. flexiblen impliziten Argumente. Flexibel implizite Argumente verhalten sich in manchen Beispielen wie definite, in anderen wiederum wie indefinite implizite Argumente (vgl. ebd. 45), (ebd. 45), vgl. (414):

(414) a. The Nobel prize is a really big deal. It's an honor just to be nominated [AWARD]. (ebd.)

b. John has been nominated [AWARD], but he still hasn't found out for which award. (ebd.)

In (414a) erhält das flexible implizite Argument eine anaphorische (d.h. definite) Interpretation, da der Kontext einen Diskursreferenten einer bestimmten Art fordert (vgl. ebd.). Dagegen erhält das implizite Argument in (414b) eine indefinite Interpretation, wie die Möglichkeit zum Sluicing deutlich macht ${ }^{239}$.

Zwar zeigen flexible implizite Argumente Merkmale definiter und indefiniter impliziter Argumente; beides gleichzeitig können sie jedoch nicht sein (vgl. ebd.).

Auch Lambrecht/Lemoine $(1996,2005)$ geben für das Französische an, dass neben definiten und indefiniten impliziten Argumenten eine weitere Art impliziter Argumente unterschieden werden muss. Ihrer Auffassung nach lassen sich drei Typen von Nichtrealisierung unterscheiden, und zwar

regardless of whether the absence of the given element is determined pragmatically or syntactically: (i) Indefinite Null-instantiation, (ii) Definite Null-instantiation, and (iii) Free-Null-instantiation. In the first, the referent of the null complement is left entirely unspecified; in the second, the referent is a specific entity or situation evoked in the speech context; in the third, the referent can be either specific or non-specific, the choice of interpretation being left up to the hearer.

(Lambrecht/Lemoine 2005: 19f.)

239 Flexible implizite Argumente stimmen mit definiten impiziten Argumenten mit Bezug auf das novelty überein (vgl. AnderBois 2012: 45). Das Kriterium der novelty basiert auf der Annahme, dass indefinite implizite Argumente neue Referenten einführen, wohingegen definit implizite Argumente dies nicht tun (vgl. ebd. 44)

Flexible implizite Argumente unterscheiden sich hingegen von definit impliziten Argumenten hinsichtlich des Sluicings (vgl. ebd. 45). Sluicing bezeichnet „the phenomenon [...] in which the crossed out material can be elided in the presence of a suitable antecedent clause in prior discourse“ (ebd.). 
Zu einem etwas anderen Ergebnis kommt Härtl (2008). Zwar weist auch er für das Deutsche auf zwei Typen intransitivierbarer Verben hin, die durch die nachfolgenden Beispiele illustriert werden:

(415) a. John brachte den Vertrag mit und Jim unterzeichnete sofort. (ebd. 23)

b. John brachte den Vertrag mit und Jim las sofort. (ebd.)

c. John brachte den Vertrag mit und Jim las ihn sofort. (ebd.)

Anders als Jacobs, Ágel, Engelberg u. a. stellt er jedoch die Annahme in Frage, wonach das nicht realisierte Thema von lesen hier eine definite Referenz annimmt und damit schließlich auch die Existenz von Verben mit definitheitsneutralen impliziten Argumenten (vgl. ebd. 117).

Seiner Auffassung nach wird nur mit einem intransitivierten Verb des Typs unterzeichnen auf das Vorhandensein eines spezifischen impliziten Arguments verwiesen. Ein solch spezifischer nominaler Ausdruck wird in dem in (415a) genannten Beispiel ,in der Kontextdomäne gegeben, weshalb der Verbalkomplex im zweiten Konjunkt so interpretiert wird, dass Jim genau den Vertrag unterzeichnet, den John mitbringt“ (ebd. 23); ein „Bezug auf eine andere, nichtvorerwähnte Entität ist nur schlecht möglich“ (ebd. 36). Anders verhält es sich dagegen bei dem in (415b) dargestellten Verb lesen. Zwar „kann sich die nicht realisierte Argumentstelle auf eine im Diskurs gegebene Entität beziehen, sie ist also hintergrundfähig“ (ebd. 117); definite Referenz nimmt sie dabei nach Härtl jedoch nicht an (vgl. ebd.). Vielmehr fokussiert lesen in seiner intransitiven Form „auf die Verbalhandlung und ihren Verlauf“ (ebd. 23) ${ }^{\mathbf{2 4 0}}$. Dabei wird in Bezug auf die Interpretation von impliziten Argumenten ,auf die Existenz eines „unmarkierten“, proto-typischen Themas verwiesen. Dies können klassische Entitäten wie etwa BUCH oder ZEITSCHRIFT sein.“ (ebd. 36) Es wird jedoch „kein Signal für eine referentielle Konstruktion des nicht ausgedrückten Themas [gegeben,

240 Auch andere Autoren weisen auf den Umstand hin, dass mit ,unterschiedlicher Perspektive auf die zu beschreibenden Vorgänge und ihre Handlungsträger [...] das Argumentpotential voll ausgeschöpft oder reduziert“ (Gansel 1993: 157) wird. Weinreich (1993) konstatiert etwa, dass der „Verzicht auf die Besetzung aller Handlungsrollen, die von der lexikalischen Valenz her zugelassen sind, [...] die Aufmerksamkeit des Hörers auf die verbleibenden Handlungsrollen und auf das Verb“ (ebd. 137) konzentrieren soll. Und auch Birk (2006: 122) schreibt, dass mit „dem elliptischen Gebrauch der Verben schreiben und helfen [...] die zum Ausdruck gebrachte Tätigkeit fokussiert wird“. 
TvdB], was - wenn es die Proposition erfordert - die Einsetzung des pronominalen Ausdrucks ihn [...] nötig macht“ (ebd. 23). Entsprechend beschreibt (415c)

eine „markierte“ Situation: Hier wird kein proto-typisches Thema gelesen, sondern die kontextuell konkrete Entität VERTRAG. Dies erfordert es, die Referenz der leer gelassenen Argumentstelle von lesen unzweideutig abzusichern: Dazu wird ein Ausdruck - das Pronomen - eingesetzt, der eine spezifische Bindung der entsprechenden Argumentstelle erreicht.

(ebd. 36)

Beispiele, in denen die Interpretation durch ein Prinzip der räumlichen Kontiguität gesteuert wird, betrachtet Härtl als elliptische Konstruktionen. Dabei wird zwar (416b) anders als (416a) bevorzugt so interpretiert, „dass der Professor genau das liest, was er in der Hand hält“ (ebd. 23):

(416) a. Der Professor druckte gerade ein Manuskript aus und las. (ebd.)

b. Der Professor hielt gerade ein Buch in der Hand und las. (ebd.)

c. Der Professor druckte gerade ein Manuskript aus und las darin. (ebd.)

Diese Interpretation resultiert jedoch ausschließlich aus der sog. räumlichen Kontiguität. Das entsprechende Prinzip besagt,

dass ein LESEN-Ereignis die Existenz eines räumlich und visuell zugänglichen und damit kognizierbaren Themas (ein Buch) erfordert. Erfüllt wird diese Vorgabe durch das im ersten Konjunkt gegebene direkte Objekt, welches durch in der Hand unzweideutig als dem Agens zugänglich identifiziert wird, was den Einsatz eines entsprechenden pronominalen Ausdrucks im zweiten Konjunkt [...] überflüssig macht (ebd), vgl. (416c).

Der Unterschied zwischen Sätzen wie (415a) und (415b) besteht nach Ansicht Härtls darin, dass es sich bei Verben wie unterzeichnen prinzipiell $-\mathrm{d}$.h. in ihrer transitiven wie intransitiven Verwendung - um telische Verben handelt, die einen Zustandswechsel beschreiben (vgl. ebd.).

Dies drückt sich in der verbalen Dekompositionsstruktur durch die Konstante BECOME aus, welche ihrerseits einen Zustand (STATE) prädiziert. Dieses Zustandsprädikat verfügt über eine Argumentstelle - das Thema -, welche obligatorisch gebunden werden muss. (ebd. 24)

Bei Verben wie lesen verhält es sich dagegen anders. Zwar können diese Verben „einen Ausdruck eines telischen und durativen Ereignisses [...] [aufspannen, TvdB], wenn das interne Argument realisiert ist“ (Härtl 2008: 120). In seiner intransitiven Verwendung handelt es sich nach Härtl aber um ein atelisches Verb, das „eine Fokussierung auf die Verbalhandlung [erlaubt, TvdB], ohne dass ein Zustandswechsel eines Themas ein in irgendeiner Form notwendiger Teil der Bedeutung sein müsste“ (ebd. 24). 
Auch Rapp ${ }^{241}$ (1997) weist ausgehend von nachfolgenden Beispielen auf diesen Umstand hin:

(417) a. Mario liest das Buch in zwei Stunden/* zwei Stunden lang. (TvdB)

b. Mario liest *in zwei Stunden/zwei Stunden lang. (ebf.)

Sie konstatiert:

Die Vollvariante bezeichnet einen Prozeß, d.h. eine durch Anfangs- und Endzustand begrenzte Entwicklungsphase; als durative Zeitangabe kann folglich nur ein Rahmenadverbial verwendet werden. Konträr die Situation bei der Fakultativitätsvariante: Die alleinige Möglichkeit des Spannenadverbials zeigt an, daß hier e-strukturell eine Tätigkeit und kein Prozeß vorliegt.

(ebd. 496)

Um diesen Vorgang zu erfassen, nimmt Rapp eine Detransitivierungsregel ${ }^{242}$ an, vgl. (418). Diese schneidet aus der (transitiven) Vollvariante den Tätigkeitsteil heraus und erzeugt dadurch eine (intransitive) Fakultativitätsvariante (vgl. ebd. 496). Durch die vollständige Löschung der internen Argumentstelle werden entsprechende Verben in ihren intransitiven Verwendungen ihrer Auffassung nach zu Tätigkeiten (vgl. ebd. 498):

$$
\text { CAUSE (DO (x), DEV (BE (y))) } \rightarrow \text { DO (x) }
$$

\section{Nach Rapp eleminiert die Detransitivierungsregel in (418) das}

als Akkusativobjekt realisierte y-Argument - dies hat zur Folge, daß die Prädikate BE, DEV und CAUSE, unter die y eingebettet ist, ebenfalls getilgt werden. Nicht betroffen ist dagegen das Prädikat DO, welches als Argument nur den als Subjekt realisierten Actor x besitzt.

(ebd.)

Damit ist ihrer Auffassung nach bei intransitiven Verbverwendungen des Typs lesen kein implizites Argument in der lexikalisch-semantischen Struktur verankert.

241 Rapp ging es in ihren Untersuchungen nicht darum, den Unterschied zwischen definiten und indefiniten bzw. definitheitsneutralen impliziten Argumenten zu erfassen; vielmehr bestand ihr Ziel darin zu zeigen, dass sich es sich bei der Fakultativität von Argumenten nicht, wie zumeist angenommen, um eine idiosynkratische Eigenschaft von Verben handelt, sondern dass sie sich ,überwiegend aus der lexikalisch-semantischen Struktur (= LSS) des Verbs ableiten läßt“ (Rapp 1997: 491).

242 Als Detransitivierung bezeichnet Rapp einen Vorgang, bei dem die lexikalisch-semantische Struktur des Verbs in spezifischer Weise verändert und das betreffende Argument vollständig gelöscht wird.

$\mathrm{Zu}$ den Verben, die eine solche Detransitivierung zulassen, gehören u. a. kausative Prozessverben wie malen und essen. Mit Blick auf Verben wie verbiegen und fällen merkt die Autorin jedoch zu Recht an, dass durchaus nicht alle kausativen Prozessverben detransitivierbar sind (vgl. ebd. 496). 
Diese Ansicht vertritt mit Verweis auf Beispiele wie in (419) auch Härtl. Bei dem dort realisierten Verb abladen handelt es sich im Unterschied zu dem zuvor dargestellten Verb lesen um ein inhärent telisches Verb, welches sowohl mit als auch ohne realisiertes Themaargument mit Hilfe eines Rahmenadverbials ${ }^{\mathbf{2 4 3}}$ modifiziert werden kann. Damit liegt hier anders als bei dem in (415b) genannten Beispiel „also tatsächlich definite Referenz eines impliziten Themas vor [...], was [...] in der Diskursdomäne gebunden wird“ (ebd.117).

(419) a. Günther fuhr den Möbelwagen vor und Peter lud ihn in zehn Minuten ab. (ebd.)

b. Günther fuhr den Möbelwagen vor und Peter lud in zehn Minuten ab.(ebd.)

Hieraus sowie aus der Beobachtung, dass „nicht realisierte Themen sich nicht auf (informationsstrukturell) saliente Antezedenten beziehen“ (ebd. 122) können, vgl. (420), kommt auch Härtl zu dem Schluss, „dass bei intransitivierten Verben [des Typs lesen, TvdB] keine Argumentposition in der lexikalisch-semantischen Repräsentation des betreffenden Ausdrucks zur Verfügung steht“ (ebd. 129).

(420) A: Hat Gerda das Buch gelesen?

B: Ja, sie las *(es).

Diese Annahme wird seines Erachtens zusätzlich dadurch gestärkt, dass „nur bei intransitiv verwendeten Activities [...] eine Annullierung eines möglichen Bezugs auf eine im Kontext gegebene Argument-Entität erlaubt ist [...]“ (ebd.), vgl. (421a); bei inhärent telischen Achievements (vgl. (421b)) und Accomplishments (vgl. (421c)) ist dies hingegen wesentlich markierter:

(421) a. Karl kaufte sich einen Bestseller und las dann, und zwar eine Zeitschrift. (ebd.)

b. John brachte den Vertrag mit und Jim unterzeichnete dann, ??und zwar ein Zeugnis. (ebd.)

c. Günther fuhr den Möbelwagen vor und Peter lud dann ab, ??und zwar eine Schubkarre. (ebd.)

243 Die Modifizierbarkeit von Verben wie unterzeichnen mit Hilfe eines Rahmenadverbials ist ebenfalls ausgeschlossen, da es sich hierbei nicht um ein Accomplishment, sondern um ein Achievement handelt. Da es sich bei Achievements - im Unterschied zu Accomplishments - um nicht durative Verben handelt, ist die Modifikation durch Zeitspannenadverbiale ausgeschlossen (vgl. Rothstein 2007). 
Die Bobachtung, wonach nicht realisierte Themen von Verben wie lesen durchaus in der Lage seien, neue Entitäten in den Diskurs einzuführen, vgl. (422a), entkräftet Härtl, indem er feststellt, dass das Pronomen es in Bridging-Konstruktionen wie (422a) zwar anaphorischen Charakter aufweist, seine Referenz aber nicht grammatisch sondern via Ereignis-Bezug konstruiert werden muss, weshalb sie sich nicht auf eine neu kreierte Diskursentität beziehen kann (vgl. ebd. 123). Gestützt wird diese Annahme durch das in (422b) genannte Beispiel, „bei welchem sich es auf die vorerwähnte Verbalprojektion als Ausdruck des Ereignisses bezieht“ (ebd.).

(422) a. Ich habe vor einer Stunde $e_{i}$ gegessen und $e_{i}$ war sehr sättigend. (ebd. 122)

b. Hannes wollte rauchen ${ }_{i}$, aber es $_{i}$ war ihm zu teuer geworden. (ebd. 123)

Auch andere Autoren zweifeln die Existenz indefinit impliziter Argumente an. So geben Junker et al. (2006) mit Verweis auf Fillmores Aussage, dass ein indefinites implizites Argument ,is obligatorily disjoint in reference with anything saliently present in the pragmatic context“ (Fillmore 1986: 97) für das Englische an, dass

a better characterization of indefiniteness is what Fillmore hints at elsewhere in the paper: the null complement is interpreted as some kind of existential quantification, hence not as reference at all. The contrast is thus not "reference to something salient, versus reference to a particular but unknown object”. At best, it is reference versus (existential) quantification.

(Junker et al. 2006: 6)

Doch auch das, so die Autoren, ist nicht ganz zutreffend: Verben mit indefinit impliziten Argumenten weisen nicht die Skopusambiguitäten auf, die man bei coverter existentieller Quantifikation erwarten würde.

Whereas [...] [No one ate something; $\mathrm{TvdB}$ ] has a reading with 'something' given wide scope there is a dish, say the spinach spaghetti with tofu balls, that no one ate - [...] [No one ate; $\mathrm{TvdB}$ ] has no such reading. [...] If we must find a synonym for [...] ]No one ate; TvdB] that has something overt in the complement spot, 'No one ate stuff' would serve better. In light of these sentences, Fillmore will need to say that INC verbs are read as "V-ed stuff"244.

(ebd. 6f.; Fußnote ergänzt, TvdB)

244 Weiter konkretisieren die Autoren, dass No one ate „doesn’t exactly mean ,No one ate something', or even ,No one ate stuff'. ,No one ate' means, rather, that no one ate“ (Junker et al. 2006: 11). 
Hiervon ausgehend reklassifizieren Junker et al. Verben, die „hartnäckig“ eine Existenzlesart aufweisen - d.h. Verben, die ursprünglich als solche mit indefiniten impliziten Argumenten klassifiziert wurden - ,as not really null complement verbs“ (ebd. 9). Danach sind Verben wie eat bereits im Lexikon als intransitiv markiert, weshalb implizite Argumente von vorneherein ausgeschlossen $\operatorname{sind}^{245}$.

\subsection{Verbübergreifende Betrachtung}

Die vorangehenden Darstellungen zeigen, dass in der Forschung keine einheitliche Linie im Zusammenhang mit definiten und indefiniten Interpretationen impliziter Argumente vertreten wird. Vor allem die Interpretation indefiniter (bzw. definitheitsneutraler) impliziter Argumente wird dabei kritisch betrachtet und zuweilen auch in Frage gestellt.

Im Folgenden wird ausgehend von der in F2 formulierten Frage sowie den in Kapitel 5 gemachten Beobachtungen für semantische Spezialisierungen impliziter Argumente folgende Hypothese vertreten: Während Sätze wie in (423) der gleichen Verblesart zugeordnet werden können, (423b) damit eine valenzreduzierte Form von (423a) darstellt, trifft diese Interpretation auf Sätze wie in (424) nicht zu. Vielmehr lassen sich die in (424a) und (424b) angegeben Verwendungsweisen des Verbs eigenständigen Lesarten mit jeweils spezifischen semantischen und syntaktischen Valenzforderungen zuordnen:

(423) a. Peter hat den Vertrag unterschrieben. (TvdB)

b. Peter hat unterschrieben. (ebf.)

(424) a. Bruno isst einen Apfel. (ebf.)

b. Bruno isst. (ebf.)

Bei Sätzen wie in (423) handelt es sich um Fälle sog. definiter Weglassungen, bei denen das weggelassene Argument von Sprecher und Hörer aus dem sprachlichen oder situativen Kontext hergeleitet werden kann (vgl. Ruppenhofer/Michaelis 2014: 60). Weglassungen dieser Art zeichnen sich nach gängiger Auffassung

245 Der Tatsache, dass Verben wie eat durchaus mit Objekt realisiert werden können, begegnen Junker et al. mit der Überlegung, dass es sich bei diesen Verben um intransitive Verben handelt, die gekennzeichnet sind „for optional addition of an argument“ (ebd. 10). 
dadurch aus, dass entsprechende Verben in ihrer transitiven wie intransitiven Verwendung telische Ereignisse beschreiben ${ }^{246}$ (vgl. Härtl 2008). D. h., sie „drücken einen Zustandswechsel von einem Ausgangszustand zu einem der Verbbedeutung inhärenten Endzustand aus“ (Rothstein 2007: 9). Überprüft werden kann die Telizität mit Hilfe des sog. XY-lang-Tests, „wobei X für eine beliebige Zahl und Y für eine beliebige Zeiteinheit stehen. Atelische Verben erlauben XY-lang, telische verbieten XY-lang. “( (ebd.):

(425) a. ^Peter hat den Vertrag drei Stunden lang unterschrieben. (TvdB)

b. ?Peter hat drei Stunden lang unterschrieben ${ }^{247}$. (ebf.)

Die intransitiven Verwendungen dieser Verben verfügen „nicht über ein arbiträres, sondern vielmehr über ein spezifisches pronominales Element in der Objekt-Position“ (Härtl 2008: 117), weshalb hier definite Referenz eines impliziten Themas vorliegt, das in der Diskursdomäne gebunden wird (s. o.). $\mathrm{Zu}$ den Verben - im Sinne der vorliegenden Arbeit in jeweils einzelnen Lesarten -, die in diesem Zusammenhang häufig genannt werden, gehören u. a. unterschreiben, akzeptieren, einwilligen und öffnen. Neben ihrer inhärenten Telizität zeichnen sie sich dadurch aus, dass die Annullierung eines möglichen Bezugs auf eine im Kontext gegebene Argument-Entität nicht erlaubt ist, vgl. (421b), wohingegen eine stattdessen-Erweiterung bei Negation problemlos möglich scheint (vgl. Glass 2014: 129), vgl. (426):

Der Vertrag war gut, doch Peter unterschrieb nicht. Er unterschrieb stattdessen eine Woche später den Vertrag bei der Konkurrenz. (TvdB)

Im Folgenden werden transitive und intransitive Verwendungen dieser Verben der gleichen Lesart zugeordnet, d.h. die argumentlosen Verwendungen werden als valenzreduzierte Formen der transitiven Realisierungen betrachtet. Für das in (423) dargestellte Verb unterschreiben ergeben sich die in (427) dargestellten Valenzen. Dabei hat die valenzreduzierte Variante ein nicht- $\lambda$-gebundenes, $d . h$. implizites Argument (vgl. Engelberg 2002: 373), vgl. (427b). Das Subskript ,+Def

246 Dabei darf m. E. nicht außer Acht gelassen werden, dass Telizität immer auch mit der Beschaffenheit des Objekts zusammenhängt (vgl. auch FN 238).

247 Eine mögliche Akzeptabilität solcher Sätze resultiert aus iterativen Lesarten, ,also Lesarten, bei denen das Ereignis wiederholt stattfindet“ (Rothstein 2007: 9). 
zeigt an, dass das implizite Relat definit ${ }^{248}$, d.h. in der Äußerungssituation bekannt sein muss (vgl. Jacobs 2003: 393):
a. unterschreiben
i.S.v. jemand unterzeichnet etwas
synVal: $/$ nom $^{\mathrm{y}} / \mathrm{akk}^{\mathrm{x}}$
semVal: $\lambda \mathrm{x} \lambda \mathrm{y}$ [UNTERSCHREIB (x) (y)]
b. unterschreiben $n_{R}$
synVal: $/$ nom $^{\mathrm{y}}$
semVal: $\lambda y\left[\right.$ [UNTERSCHREIB $\left.{ }_{\mathrm{R}}\left({ }_{+\mathrm{Def}} \mathrm{X}\right)(\mathrm{y})\right]$

Die enge semantische Verbindung beider Verbverwendungen kann durch eine Bedeutungsregel erfasst werden:

(428) BR: $\forall x \forall y\left[\operatorname{UNTERSCHREIB}_{\mathrm{R}}(\mathrm{x})(\mathrm{y}) \rightarrow \operatorname{UNTERSCHREIB~}(\mathrm{x})(\mathrm{y})\right]$

Bei Sätzen wie in (424) handelt es sich um Verwendungsweisen, die bislang unter der Bezeichnung indefinite bzw. definitheitsneutrale Weglassungen zusammengefasst wurden. Genannt werden in diesem Zusammenhang i.d.R. Verben wie essen, trinken, lesen, malen und singen.

Nach gängigen Analysen drücken diese Verben in ihrer transitiven Verwendung einen Zustandswechsel von einem Ausgangszustand zu einem Endzustand aus und beschreiben somit telische Ereignisse. Hingegen weisen sie in ihrer intransitiven Verwendung keinen natürlichen Endzustand auf; sie beschreiben dementsprechend atelische Aktivitäten und erlauben damit eine Fokussierung auf die Verbalhandlung (vgl. u. a. Rapp 1997; Rappaport Hovav/Levin 1998; Härtl 2008) - so ist z.B. argumentlos verwendetes essen ein atelisches Verb, da das Verb lexikalisch keinen Endzustand ausdrückt; nur unser Weltwissen sagt, dass man nicht ewig essen kann (vgl. Rothstein 2007: 9). Auch hier - so die gängige Auffassung - kann die Telizität bzw. Atelizität mit Hilfe des XY-lang-Tests überprüft werden:

(429) a. 'Bruno isst drei Stunden lang einen Apfel. (iterativ)

b. Bruno isst drei Stunden lang.

248 Jacobs (1994b) nimmt für solche Fälle definite Variablen („x“, „y“, „z“) an, „die, wenn sie frei vorkommen, einen in den Diskurs bereits eingeführten Referenten haben müssen“ (ebd. 300). Sie sind von definitheitsneutralen Variablen (,u“, „,““, „,w“) zu unterscheiden, die die Vorerwähntheit eines Referenten im Diskurs weder fordern noch verbieten: $\lambda y\left[\operatorname{HEIRAT}_{\mathrm{R}}(\mathrm{u})(\mathrm{y})\right]$ (vgl. ebd. 298). 
Eine solche Analyse ist m. E. aus unterschiedlichen Gründen nicht unproblematisch. Zum einen ergibt sich die (Nicht-)Telizität entsprechender Verben nicht aus ihrer (In-)Transitivität; vielmehr ist sie auf die Beschaffenheit der Objekt-NP zurückzuführen. So entsteht die Telizität von Bruno isst einen Apfel in (429a) nicht durch die vorliegende Transitivität, sondern aus der Gequanteltheit eines inkrementellen Arguments. Liegt diese Quantelung nicht vor, tritt auch in der transitiven Variante keine Telizität auf ${ }^{249}$, vgl. (430):

(430) Bruno isst drei Stunden lang Äpfel.

Zum anderen offenbart sich bei Verben wie heiraten, sich verloben und sich scheiden lassen, die ebenfalls im Zusammenhang mit indefiniter bzw. definitheitsneutraler Weglassung diskutiert werden, ein weiteres Problem. Anders als die zuvor genannten Verben essen, trinken und lesen handelt es sich bei ihnen nicht um Aktivitäten i.e.S. Entsprechend sind Sätze wie die nachfolgenden nicht möglich ${ }^{250}$ :

(431) a. *Sie ließ sich zwei Tage lang scheiden.

b. ^Er heiratete zwei Tage lang.

c. ${ }^{\star}$ Sie verlobten sich zwei Tage lang.

Um diesen Beobachtungen gerecht $\mathrm{zu}$ werden, wird nachfolgend davon ausgegangen, dass bei Verben, die im Zusammenhang mit indefiniter bzw. definitheitsneutraler Weglassung diskutiert werden, die transitiven und intransitiven Verwendungen jeweils unterschiedlichen Lesarten zuzuordnen sind. Beide Lesarten gehen dabei mit je spezifischen Profilierungen (vgl. Goldberg 1995) einher, wobei hier der Ausdruck Profilierung ,auf den Umstand, dass obligatorische Argumente semantisch hervorgehoben, in diesem Sinne also profiliert bzw. perspektiviert [...] sind“ (Welke 2019: 217), zielt. Während entsprechende Verben in ihren transitiven Verwendungen das, was gegessen, getrunken oder gelesen wird bzw. wer geheiratet oder sich verlobt hat fokussieren, stehen bei ihren intransitiven Verwendungen die vom Verb ausgedrückten Handlungsformen als solche -

249 Vor dem Hintergrund, dass auch bei der zuvor dargestellten definiten Weglassung die Beschaffenheit der Objekt-NP Einfluss auf die Telizität hat - so liegt auch hier bei einem nicht gequantelten Argument keine Telizität vor (vgl. Peter unterschreibt drei Stunden lang Verträge) -, stellt sich m. E. die Frage, inwieweit die Unterscheidung zwischen definiter und indefiniter Weglassung tatsächlich etwas mit Telizität zu tun hat.

250 Danach hat die Unterscheidung zwischen definiter und indefiniter Weglassung unter Umständen weder etwas mit Telizität noch mit Aktivität zu tun. 
also das Essen, Trinken und Lesen bzw. das Heiraten und sich Verloben - im Vordergrund.

Die unterschiedlichen Lesarten der entsprechenden Verben sind mit je spezifischen semantischen und syntaktischen Valenzen verbunden, die die Besonderheiten der Verwendungsweisen wiederspiegeln, vgl. exemplarisch für das Verb essen $^{251}$ (432) und (433):

(432) essen $_{1}$

i.S.v. jemand nimmt (irgendetwas als) feste Nahrung zu sich

synVal: $/$ nom $^{\mathrm{x}}$

semVa: $\lambda x\left[\operatorname{ESS}_{1}(\mathrm{x})\right]$

(433) essen $_{2}$

i.S.v. jemand verzehrt etwas Bestimmtes als Nahrung

synVal: $\quad /$ nom $^{\mathrm{y}} / \mathrm{akk}^{\mathrm{x}}$

semVal: $\lambda x \lambda y\left[\operatorname{ESS}_{2}(\mathrm{x})(\mathrm{y})\right]$

„Die Wahl eines bestimmten Valenzträgers bedeutet somit zugleich die Perspektivierung eines ganzen Skripts“ (Nikula 2007: 205): essen (432) beschreibt Situationen, in denen jemand dabei ist, irgendetwas (als feste Nahrung/Nahrungsmittel) zu sich zu nehmen. Dabei geht es nicht in erster Linie darum zu benennen, was jemand isst, sondern vielmehr darum, den Verbalvorgang als solchen - hier das Essen - zu fokussieren. In diesem Sinne ist nur eine semantische Rolle in der entsprechenden Verbbedeutung vorangelegt, und zwar die desjenigen, der die Handlung des Essens ausführt. Sie kann über die Verursacher-Relation auch als Agens klassifiziert werden. essen $_{2}$ (433) dagegen beschreibt Situationen, in denen jemand etwas Bestimmtes als Nahrung verzehrt. Dabei wird - anders als bei essen $n_{1}$ - nicht der essenVorgang als solcher in den Fokus gestellt; vielmehr wird das, was gegessen wird, hervorgehoben. Entsprechend sind bei essen 2 zwei semantische Rollen bedeutungskonstitutiv: Zum einen derjenige, der den Vorgang des Essens ausführt, und zum anderen dasjenige, das von dem essen-Vorgang betroffen ist. Während der erste Partizipant über die Verursacher-Relation auch als Agens klassifiziert wird, kann der zweite Partizipant über die Relation der Betroffenheit/Veränderung als Patiens eingestuft werden.

251 Bereits Fodor/Fodor (1980) merkten für das Verb eat an, dass „there are several predicates eat, eat', eat", and so on. Each of these predicates has a different (but fixed) number of functional argument positions. In fact, each of these predicates has exactly as many functional argument positions as it has surface syntactic argument positions. Meaning postulates capture the interrelations between sentences which contain these predicates“ (ebd. 764). 
Zwar weisen klassische Tests zum Nachweis einer Implikatur darauf hin, dass essen $_{1}$ ein implizites Thema in seinen Wahrheitsbedingungen enthält (vgl. Härtl 2008: 121):

(434) \#Bruno isst, aber es gibt nichts, was er isst.

Dass es sich dabei jedoch nicht um eine Argumentposition in der lexikalischsemantischen Repräsentation des Verbs handelt, konnte u.a. von Härtl (2008) gezeigt werden - vgl. etwa die Unmöglichkeit, dass sich nicht realisierte Themen auf (informationsstrukturell) saliente Antezedenten beziehen (Hat Bruno den Apfel gegessen? - ${ }^{\star}$ Ja, er hat gegessen.). Dass bei den intransitiven Verwendungen dieser Verben keine definite Referenz vorliegt, zeigen auch die Beispiele (419b) und (435): Während bei intransitiv verwendetem essen eine Modifikation mit einem Rahmenadverbial ausgeschlossen ist (435), ist sie bei inhärent telischen Verben wie abladen ohne weiteres möglich (419b). Damit liegt bei abladen im Unterschied zu essen tatsächlich definite Referenz eines impliziten Themas vor (vgl. Härtl 2008: 117):

$$
\text { ^Bruno isst in zehn Minuten. }(\mathrm{TvdB})^{252}
$$

Darüber hinaus zeigt sich, dass bei entsprechenden Verben die Annullierung eines möglichen Bezugs auf eine im Kontext gegebene Argument-Entität problemlos möglich ist, vgl. (421a), wohingegen eine stattdessen-Erweiterung bei Negation ausgeschlossen scheint (vgl. Glass 2014: 129), vgl. (436):

(436) There was bread on the table but John didn't eat. ??He ate grapes instead. (nach Glass 2014: 129)

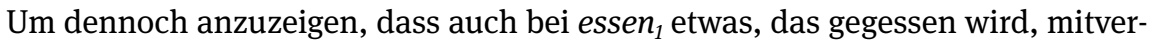
standen wird - so kann niemand die Tätigkeit des Essens im Sinne von essen ausführen, ohne dabei im Sinne von essen $_{2}$ etwas zu essen (s. u.) -, kann die in (437) dargestellte Bedeutungsregel angenommen werden. Sie erfasst die enge Verbindung zwischen beiden essen-Lesarten und gibt auch an, warum es sich bei (434) um eine Kontradiktion handelt:

$$
\text { BR: } \forall \mathrm{x}\left[\mathrm{ESS}_{1}(\mathrm{x}) \rightarrow \exists \mathrm{y}\left[\mathrm{ESS}_{2}(\mathrm{x})(\mathrm{y})\right]\right.
$$

252 Für manche Sprecher ist diese Verbverwendung i.S.v. das Essen abschließen/durchführen akzeptabel; der eigenen Intuition entspricht sie eher nicht. 
Folgt man der o.g. Hypothese, können auch die in Kapitel 5.1.2.5 angestellten Überlegungen zum Verb trinken unter Umständen modifiziert werden: Das dort dargestellte Verb trinken ${ }_{1}$ beschreibt Situationen, in denen jemand etwas als Getränk zu sich nimmt, vgl. (226) hier wiederholt in (438). Dabei sind zwei semantische Rollen in der Bedeutung des Verbs vorangelegt: Derjenige, der die Tätigkeit des Trinkens ausführt und über die Verursacher-Relation auch als Agens klassifiziert werden kann, und dasjenige, dass von dem trinken-Vorgang betroffen ist und eine Zustandsveränderung erfährt, somit über die Relation der Betroffenheit/Veränderung als Patiens eingestuft werden kann.

(438) a. Opa Hermann sitzt auf der Couch und trinkt Kaffee.

b. Monikas Baby trinkt Milch aus seinem Fläschchen.

c. Felix, die Katze der Nachbarn, trinkt Wasser aus unserem Teich.

trinken $_{1}$ weist entsprechend die folgenden semantischen und syntaktischen Valenzen auf, vgl. (225a) hier wiederholt in (439):

(439) trinken $_{1}$

i.S.v. jemand verzehrt etwas als Getränk

synVal: $/$ nom $^{\mathrm{y}} / \mathrm{akk}^{\mathrm{x}}$

semVa: $\lambda x \lambda y\left[\operatorname{TRINK}_{1}(\mathrm{x})(\mathrm{y})\right]$

BR: $\quad \forall x \forall y$ [TRINK $_{1}(\mathrm{x})(\mathrm{y}) \rightarrow \operatorname{LEBEWESEN}(\mathrm{y}) \&$ FLÜSSIGKEIT(x)]

Abweichend $\mathrm{zu}$ der Darstellung in Kapitel 5.1.2.5, nach der trinken Komple- $^{-}$ mentweglassung erlaubt und damit eine entsprechende R-Valenz anzunehmen ist (vgl. trinken ${ }_{1 \_R}$ in (228b)), kann man auf der hier entwickelten Linie schlussfolgern, dass transitive Verwendungen wie in (438) einer eigenständigen Lesart des Verbs trinken zuzuordnen sind. Im Unterschied $\mathrm{zu}$ trinken $_{1}$ beschreibt die im weiteren Verlauf dann als trinken $_{4} \mathrm{zu}$ bezeichnende Verbverwendung Situationen, in denen jemand dabei ist, ein Getränk zu sich zu nehmen. Dabei geht es nicht in erster Linie darum, genauer zu spezifizieren, was getrunken wird, sondern vielmehr darum, den Trinkvorgang als solchen genauer zu beschreiben:

(440) a. Opa Hermann sitzt auf der Couch und trinkt.

b. Monikas Baby trinkt.

c. Felix, die Katze der Nachbarn, trinkt.

In diesem Sinne ist nur eine semantische Rolle in der entsprechenden Verbbedeutung vorangelegt, und zwar die desjenigen, der die Handlung des Trinkens 
ausführt; sie wird über die Verursacher-Relation auch als Agens klassifiziert. trinken $_{4}$ weist entsprechend die in (441) dargestellte semantische und syntaktische Valenz auf:

(441) trinken $_{4}$

i.S.v. jemand nimmt ein Getränk zu sich

synVal: $/$ nom $^{\mathrm{x}}$

semVl: $\quad \lambda x\left[\operatorname{TRINK}_{4}(\mathrm{x})\right]$

Eine solche Analyse trifft auf die in (232) genannten und in (442) wiederholten Beispiele ebenfalls zu; auch hier wird die Handlungsform des Trinkens als solche - hier vor allem in Opposition zum Nicht-Trinken - fokussiert. Nicht konkretisiert werden dagegen die Flüssigkeiten, die getrunken werden:

(442) a. Ich trinke nie auf langen Autofahrten.

b. Du kannst ruhig trinken - wir sind gleich da.

c. Hast du getrunken? Du weißt, dass du bei diesen Temperaturen viel trinken musst.

Die enge Verbindung der beiden Lesarten trinken ${ }_{1}$ und trinken 4 kann auch hier durch eine entsprechende Bedeutungsregel erfasst werden:

(443) BR: $\forall x\left[\operatorname{TRINK}_{4}(\mathrm{x}) \rightarrow \exists \mathrm{y}\left[\operatorname{TRINK}_{1}(\mathrm{x})(\mathrm{y})\right]\right.$

Die vorgeschlagene Analyse steht nicht im Widerspruch zu der von Rapp (1997) vertretenen Auffassung, wonach bei intransitiven Verwendungen von Verben des Typs malen, essen und trinken durch eine lexikalische Regel der Tätigkeitsteil aus der verbalen Vollvariante „herausgeschnitten“ wird. Dabei wird ihrer Auffassung nach die interne Argumentstelle vollständig gelöscht; entsprechend steht auch nach ihrer Analyse keine Argumentposition für das nicht-realisierte Thema in der lexikalisch-semantischen Repräsentation der so erzeugten Fakultativitätsvariante zur Verfügung (vgl. ausführlich die Darstellungen in Kapitel 6.1).

Während Rapp jedoch davon ausgeht, dass die intransitiven Verbverwendungen aus einer Art Detransitivierung und damit aus Argumentweglassung resultieren, wird in der vorliegenden Arbeit die Annahme vertreten, dass die unterschiedlichen Perspektivierungen entsprechender Verben im Sinne von F2 auf das Vorliegen unterschiedlicher Lesarten bereits vor Argumentweglassung zurückzuführen sind. 